\title{
Overexpression of a Rice NPR1 Homolog Leads to Constitutive Activation of Defense Response and Hypersensitivity to Light
}

\author{
Mawsheng Chern, ${ }^{1}$ Heather A. Fitzgerald, ${ }^{1}$ Patrick E. Canlas, ${ }^{1}$ Duroy A. Navarre,${ }^{2}$ and \\ Pamela C. Ronald ${ }^{1}$ \\ ${ }^{1}$ Department of Plant Pathology, University of California, Davis 95616, U.S.A.; ${ }^{2}$ United States Department of Agriculture- \\ Agricultural Research Service, Prosser, WA 99350, U.S.A.
}

Submitted 11 October 2004. Accepted 10 January 2005.

\begin{abstract}
Arabidopsis NPR1/NIM1 is a key regulator of systemic acquired resistance (SAR), which confers lasting broad-spectrum resistance. Previous reports indicate that rice has a disease-resistance pathway similar to the Arabidopsis SAR pathway. Here we report the isolation and characterization of a rice $N P R 1$ homologue $(\mathrm{NH1})$. Transgenic rice plants overexpressing $\mathrm{NHI}$ (NH1ox) acquire high levels of resistance to Xanthomonas oryzae pv. oryzae. The resistance phenotype is heritable and correlates with the presence of the transgene and reduced bacterial growth. Northern analysis shows that NH1ox rice spontaneously activates defense genes, contrasting with NPR1-overexpressing Arabidopsis, where defense genes are not activated until induction. Wildtype NH1, but not a point mutant corresponding to npr1-1, interacts strongly with the rice transcription factor rTGA2.2 in yeast two-hybrid. Greenhouse-grown NH1ox plants develop lesion-mimic spots on leaves at preflowering stage although no other developmental effects are observed. However, when grown in growth chambers (GCs) under low light, NH1ox plants are dwarfed, indicating elevated sensitivity to light. The GC-grown NH1ox plants show much higher salicylic acid (SA) levels than the wild type, whereas greenhouse-grown NH1ox plants contain lower SA. These results indicate that $\mathrm{NH1}$ may be involved in the regulation of $\mathrm{SA}$ in response to environmental changes.
\end{abstract}

Systemic acquired resistance (SAR) is a long-lasting plantdefense response that confers broad-spectrum resistance to viral, bacterial, and fungal pathogens and induces expression of pathogenesis-related $(P R)$ genes (Ryals et al. 1996). In dicots, such as Arabidopsis and tobacco, salicylic acid (SA) and the synthetic chemicals 2,6-dichloroisonicotinic acid (INA) and benzothiadiazole (BTH) are potent inducers of SAR (Friedrich et al. 1996). In monocots, SAR has been described for rice (Smith and Metraux 1991) and wheat (Gorlach et al. 1996) at least; and BTH has been shown to induce SAR in wheat (Gorlach et al. 1996) and disease resistance in rice (Rohilla et al. 2002; Schweizer et al. 1999) and maize (Morris et al. 1998).

Corresponding author: Pamela Ronald; E-mail: pcronald@ucdavis.edu

Current address of H. A. Fitzgerald: Department of Botany and Plant Pathology, Oregon State University, Corvallis, OR 97330, U.S.A.

Nucleotide sequence data is available in the GenBank database under accession numbers AY923983 for $\mathrm{NH} 1$ and AY923984 for NH2.
The NPRI (also known as NIMI and SAII) gene is a key regulator of SA-mediated SAR in Arabidopsis (Cao et al. 1994; Delaney et al. 1995; Glazebrook et al. 1996; Ryals et al. 1997; Shah et al. 1997). Upon induction by SA, INA, or BTH, NPRI expression levels are elevated (Cao et al. 1997; Ryals et al. 1997). NPRl affects the SAR pathway downstream of the SA signal. Arabidopsis nprl/niml mutants are impaired in their ability to induce $P R$ gene expression and mount a SAR response even after treatment with SA or INA. NPRI also is involved in the SA-promoted basal thermotolerance in Arabidopsis (Clarke et al. 2004). NPRl encodes a novel protein with a bipartite nuclear localization sequence and two potential protein-protein interaction domains: an ankyrin repeat domain and a BTB/POZ domain (Cao et al. 1997). Nuclear localization of NPR1 protein is essential for its function (Kinkema et al. 2000). Under uninduced states, NPR1 protein forms an oligomer and is excluded from the nucleus. Upon SAR induction, monomeric NPR1 emerges through redox changes, accumulates in the nucleus, and activates $P R$ gene expression (Mou et al. 2003).

Overexpression of NPRI in Arabidopsis leads to enhanced disease resistance to both bacterial and oomycete pathogens in a dose-dependent manner (Cao et al. 1998). Similarly, overexpression of Arabidopsis NPRl in rice results in enhanced resistance to pathogen Xanthomonas oryzae pv. oryzae (Chern et al. 2001), indicating the presence of a similar defense pathway in rice. Although transgenic Arabidopsis plants overexpressing NPRl acquire enhanced sensitivity to SA and BTH (Freidrich et al. 2001), they display no obvious detrimental morphological changes and do not have elevated $P R$ gene expression until activated by inducers or by infection of pathogens (Cao et al. 1998). However, in rice, overexpression of Arabidopsis NPRl potentiates a BTH- and low-light-environment-induced lesion mimic or cell death (LMD) phenotype (Fitzgerald et al. 2004).

In addition to SA, jasmonic acid (JA) and ethylene are wellstudied signals that regulate distinct defense pathways (Turner et al. 2002). Cross talk between SA- and JA-mediated pathways has been well documented (Dong 1998; Glazebrook 2001; Kunkel and Brooks 2002). The function of NPRI also is essential for JA- and ethylene-regulated, SA-independent induced systemic resistance (ISR) (Pieterse et al. 1998). NPR1 also appears to modulate the cross talk between SA- and JAdependent pathways; the antagonistic effect of SA on JA signaling requires NPR1, but not nuclear localization of the NPR1 protein (Spoel et al. 2003). The plant-specific transcription factor WRKY70 is identified as a common component in 
SA- and JA-mediated signal pathways; overexpression of WRKY70 activates SA-induced $P R$ genes, whereas antisense suppression results in activation of JA-responsive genes ( $\mathrm{Li}$ et al. 2004). WRKY70 expression is activated by SA and repressed by JA; functional $N P R 1$ is required for full-scale induction of WRKY70 expression. Epistasis analysis suggests that WRKY70 is downstream of NPR1 in the SA signal pathway.

In Arabidopsis, NPR1 differentially interacts with the Arabidopsis TGA family members of basic-region leucine zipper (bZIP) transcription factors (Despres et al. 2000; Zhang et al. 1999; Zhou et al. 2000). Among the Arabidopsis TGA members, NPR1 preferentially interacts with TGA2 (also known as AHBP-1b), TGA3, TGA5, and TGA6 (Zhang et al. 1999; Zhou et al. 2000). The ankyrin repeats of NPR1 are necessary and sufficient for the interaction, although high-affinity interactions also require the N-terminal one third of NPR1 (Zhang et al. 1999). The interaction is abolished by nprl-1 (carrying point mutation in the ankyrin repeats domain) and nprl-2 (carrying point mutation in the $\mathrm{N}$-terminal domain) mutants (Zhang et al. 1999). The interaction between NPR1 and TGA proteins facilitates binding of the TGA proteins to the SAresponsive as- 1 DNA element of the CaMV 35S promoter and the LS5 and LS7 elements of the PRI promoter (Despres et al. 2000). A GAL4:TGA2 fusion protein, consisting of GAL4 DNA binding domain and Arabidopsis TGA2, confers NPRIdependent activation of a promoter containing GAL4 binding sites (Fan and Dong 2002), suggesting in vivo interaction between NPR1 and TGA2. The binding of NPR1 to Arabidopsis TGA transcription factors may facilitate TGA protein binding to SA-responsive elements (Despres et al. 2000; Johnson et al. 2003), activate the transcription factors, or recruit the TGA transcription factor to its functional location in the nucleus. The triple knockout mutant tga2tga5tga6, but not single or double knockouts, blocked induction of $P R$ gene expression and pathogen resistance, showing an essential but redundant role of these transcription factors in SAR (Zhang et al. 2003). In rice, TGA2-like transcription factors interact with Arabidopsis NPR1 in a manner similar to Arabidopsis TGA2 (Chern et al. 2001). In summary, it has become clear that TGA proteins serve as a bridge between NPR1 and $P R$ gene induction.

A disease resistance response often is accompanied by a hypersensitive response (HR), characterized by the rapid death of plant cells around the infection site (Mysore and Ryu 2004). In Arabidopsis, at least 37 spontaneous LMD mutants have been identified that exhibit misregulation in cell death and whose phenotypes resemble pathogen-inducible, HR cell death. Many of these LMD mutants display altered defense responses and, therefore, provide a direct link between programmed cell death and defense responses in plants (Lorrain et al. 2003). For example, $c p r$ mutations, which result in constitutive expression of defense genes, cause spontaneous cell death. Similarly, mutations affecting salicylate-dependent signaling, such as ssi mutations (which suppress insensitivity to salicylate), also cause constitutive expression of defense genes and spontaneous lesions. In rice, lesion mimic mutants also have been identified that display activated expression of defense genes (Yin et al. 2000). The phenotypes of these mutants often resemble the $\mathrm{HR}$, suggesting that the LMD results in activation of defense gene expression.

Here, we report the isolation and characterization of two rice cDNA clones encoding proteins similar to Arabidopsis NPR1. Transgenic rice plants overexpressing one of the cDNA clones display enhanced resistance to $X$. oryzae pv. oryzae. Growth stage-dependent lesion-mimic phenotype and constitutive activation of defense gene expression were observed in the transgenic plants, marking a difference in regulation of defense activation between rice and Arabidopsis.

\section{RESULTS}

Isolation of two NPR1 homologs from rice.

We reported (Chern et al. 2001) isolation of rice cDNA clones coding for four groups of proteins that interact with the Arabidopsis NPR1 in yeast-two hybrid screens. One of these, NRR (GenBank accession number AY846391), was used as bait to back-screen the rice cDNA library by the yeast twohybrid method to search for rice NPR1. Characterization of NRR will be reported elsewhere.

We isolated two rice cDNA clones encoding NPR1 homologues (NH1 and NH2) after screening through approximately 16 million yeast clones with NRR as the bait. The NH1 cDNA appears to encode a full-length protein. The NH2 cDNA was apparently a truncated clone; the full-length $\mathrm{NH} 2$ protein sequence later was retrieved from the GenBank database (gi:34909872). A blast search (BLASTP) on the GenBank database with NH1 protein sequence gave a tobacco NPR1like protein (gi:21552981) (Liu et al. 2002) as the highest hit and Arabidopsis NPR1/NIM1 in third. The rice NH1 (582 amino acids [aa]) and $\mathrm{NH} 2$ (635 aa) protein sequences were aligned with Arabidopsis NPR1 (593 aa) and the tobacco NPR1-like protein (588 aa) in Figure 1 by using the Pileup program of the Wisconsin GCG and SeqWeb package. Amino acids conserved among two or more of the proteins are highlighted in bold for recognition. Amino acids crucial for the NPR1 function as defined by genetic mutants, such as npr1-1, npr1-2, and nim1-4 (marked by arrowheads), are conserved.

By pairwise comparison using the Bestfit program, we found that rice NH1 shares with the tobacco NPR1-like protein $61 \%$ identity and $73 \%$ similarity; it shares $49 \%$ identity and $60 \%$ similarity with Arabidopsis NPR1. Rice NH2 is most similar to an Arabidopsis NPR1-like protein (gi:30694701), sharing 54\% identity and 64\% similarity. NH2 shares 48, 48, and $41 \%$ identity with NH1, the tobacco NPR1-like protein, and Arabidopsis NPR1, respectively. In addition to NH1 and NH2, two members (gi:50917587 and gi:50904471) in the NH1 family were identified using NH1 to BLASTP-search the GenBank database; the former shares $42 \%$ identity and the latter shares $31 \%$ identity with NH1. Using the Arabidopsis NPR1 protein to BLASTP-search the database, NH1 appears as the top hit in rice. Thus, the NH1 protein is most likely the rice ortholog of Arabidopsis NPR1.

\section{Overexpression of rice $\mathrm{NH1}$ confers enhanced resistance to $X$. oryzae pv. oryzae.}

To test whether NH1 functions similarly to NPR1, we generated 32 independent transgenic lines overexpressing $\mathrm{NHI}$ (NH1ox) using a maize ubiquitin promoter in rice cv. LiaoGeng (LG). Six-week-old T0 transgenic plants and the LG control were challenged with $X$. oryzae pv. oryzae Korean race 1 (KR1), a highly virulent strain that overcomes Xa21-mediated resistance.

Inoculation data were collected from five independent experiments, each with its own LG control, because these plants were regenerated at different times (Fig. 2A). After inoculation with KR1, 10 lines (lines 11, 54, 58, 69, 85, 49, 63, 75, 56, and 57 ) of the 32 independently transformed NH1ox lines exhibited high levels of resistance to KR1 with lesion lengths 5.5 to $30 \%$ of the LG control. In all, 11 lines exhibited moderately enhanced resistance with leaf lesion lengths at least $40 \%$ shorter than the LG control. Lesion lengths of the remaining 11 lines (34\%) were no different than the LG control, possibly because of chromosomal position effects.

\section{Cosegregation of resistance with the NH1ox transgene.}

To further characterize the NH1ox transgenic rice, we analyzed the progeny of four independent lines to see if the en- 
hanced resistance phenotype correlated with the presence of the $U b i-N H 1$ transgene. Leaf samples from individual progeny were collected and the polymerase chain reaction (PCR) performed using one primer specific to the ubiquitin promoter and the other specific to $\mathrm{NHI}$ to determine whether the individual progeny carried the transgene. These progeny were inoculated with KR1 at 6 weeks old. Inoculation and PCR results were compared. Typical results from progeny of two independent lines (lines 11 and 54) are presented in Figure 2B. Results from the other two lines were similar. These data demonstrate co-segregation of resistance and the NH1ox transgene, supporting the hypothesis that overexpression of $\mathrm{NHI}$ leads to the resistance phenotype.

\section{Reduced bacterial populations correlate with shorter leaf lesion lengths.}

We performed bacterial growth curves analysis to test whether reduced lesion lengths correlated with lower bacterial populations. Homozygous progeny of line 11 transgenic NH1ox plants and the LG control were inoculated with KR1. Four leaves from each were measured to obtain an average value for lesion length and each leaf ground up for $X$. oryzae pv. oryzae population assessment at $0,4,8,12$, and 16 days after $X$. oryzae pv. oryzae inoculation.

Figure 3 presents results of the bacterial growth curve analysis (panel A) and leaf lesion development (panel B). No measurable leaf lesions developed till day 4 , where bacterial populations had reached over $10^{7} \mathrm{PFU} /$ leaf in both NH1ox and LG control. Leaf lesions started to develop thereafter. The $X$. oryzae pv. oryzae populations in NH1ox plants stayed relatively constant and below 108 PFU/leaf from day 4 to day 16, whereas those in the LG control continued growing, reaching over $10^{9}$ PFU/leaf. The $X$. oryzae pv. oryzae population in the LG control was 40 times larger than in the NH1ox line at day 16. Results (Fig. 3B) confirm the rapid and severe lesion development in LG and resistance in NH1ox plants described above. The reduced $X$. oryzae pv. oryzae growth in NH1ox plants was not due to smaller leaf size because these plants were no different from the LG control in their plant and leaf sizes when grown in the greenhouse, and only expanded leaves of nearly mature (6-week-old) plants were inoculated with $X$. oryzae pv. oryzae. Expanded leaves of NH1ox and LG control plants did not grow significantly differently during the incubation period after $X$. oryzae pv. oryzae inoculation. In summary, the shorter lesion

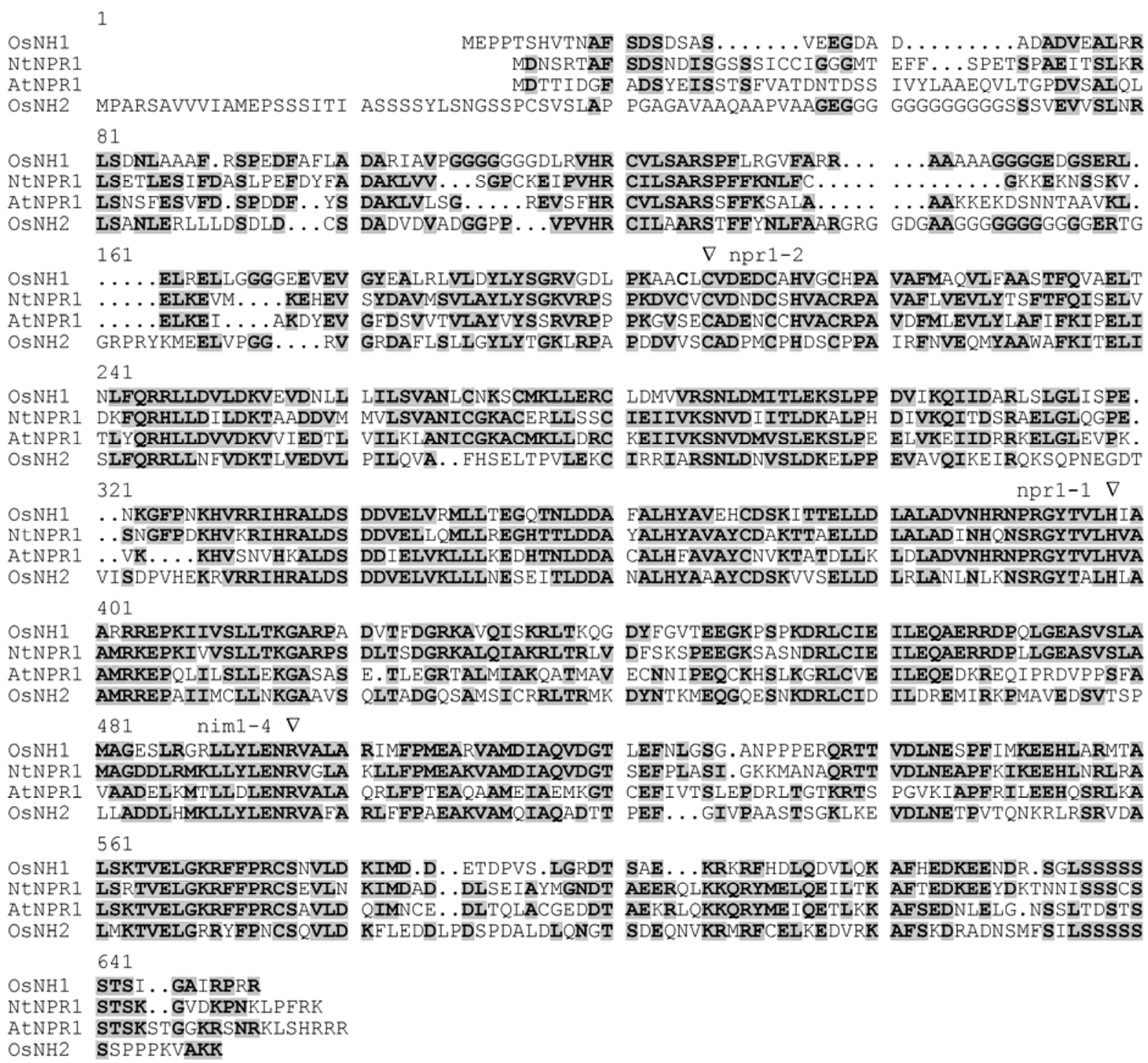

OSNH1 ARRREPKIIVSLLTKGARPA DVTFDGRKAVQISKRLTKQG DYFGVTEEGKPSPKDRLCIE ILEQAERRDPQLGEASVSLA NTNPR1 AMRKEPKIVVSLLTKGARPS DLTSDGRKALQIAKRLTRLV DFSKSPEEGKSASNDRLCIE ILEQAERRDPLLGEASVSLA ATNPR1 AMRKEPQLILSLLEKGASAS E.TLEGRTALMIAKQATMAV ECNNIPEQCKHSLKGRLCVE ILEQEDKREQIPRDVPPSFA OSNH2 AMRREPAIIMCLLNKGAAVS QLTADGQSAMSICRRLTRMK DYNTKMEQGQESNKDRLCID ILDREMIRKPMAVEDSVTSP 481 nim1-4 $\nabla$

OSNH1 MAGESLRGRLLYLENRVALA RIMFPMEARVAMDIAQVDGT LEFNLGSG. ANPPPERQRTT VDLNESPFIMKEEHLARMTA NTNPR1 MAGDDLRMKLLYLENRVGLA KLLFPMEAKVAMDIAQVDGT SEFPLASI.GKKMANAQRTT VDLNEAPFKIKEEHLNRLRA ATNPR1 VAADELKMTLLDLENRVALA QRLFPTEAQAAMEIAEMKGT CEFIVTSLEPDRLTGTKRTS PGVKIAPFRILEEHQSRLKA OSNH2 LLADDLHMKLLYLENRVAFA RLFFPAEAKVAM IAQADTT PEF. . GIVPAASTSGKLKE VDLNETPVTQNKRLRSRVDA 561

OSNH1 LSKTVELGKRFFPRCSNVLD KIMD.D..ETDPVS.LGRDT SAE... KRKRFHDLQDVLOK AFHEDKEENDR.SGLSSSSS NTNPR1 LSRTVELGKRFFPRCSEVLN KIMDAD. .DLSEIAYMGNDT AEERQLKKQRYMELQEILTK AFTEDKEEYDKTNNISSSCS ATNPR1 LSKTVELGKRFFPRCSAVLD QIMNCE. .DLTQLACGEDDT AEKRLQKKQRYMEIQETLKK AFSEDNLELG. NSSLTDSTS OSNH2 LMKTVELGRRYFPNCSQVLD KFLEDDLPDSPDALDLQNGT SDEQNVKRMRFCELKEDVRK AFSKDRADNSMFSILSSSSS 641

OSNH1 STSI. . GAIRPRR

NTNPR1 STSK. .GVDKPNKLPFRK

A.TNPR1 STSKSTGGKRSNRKLSHRRR

OSNH2 SSPPPKVAKK

Fig. 1. Sequence alignment of rice NPR1 homologues 1 (osNH1) and 2 (osNH2), Arabidopsis NPR1, and a tobacco NPR1-like protein. OsNH1, osNH2, Arabidopsis NPR1, and the tobacco NPR1-like protein (gi:21552981) were aligned using the Pileup program of the Wisconsin GCG and WebSeq package (version 2). Amino acids conserved among two or more of the proteins are highlighted in boldface. Gaps are represented by dots. The amino acids changed in npr1-1, npr1-2, and nim1-4 mutants are marked by arrowheads. 

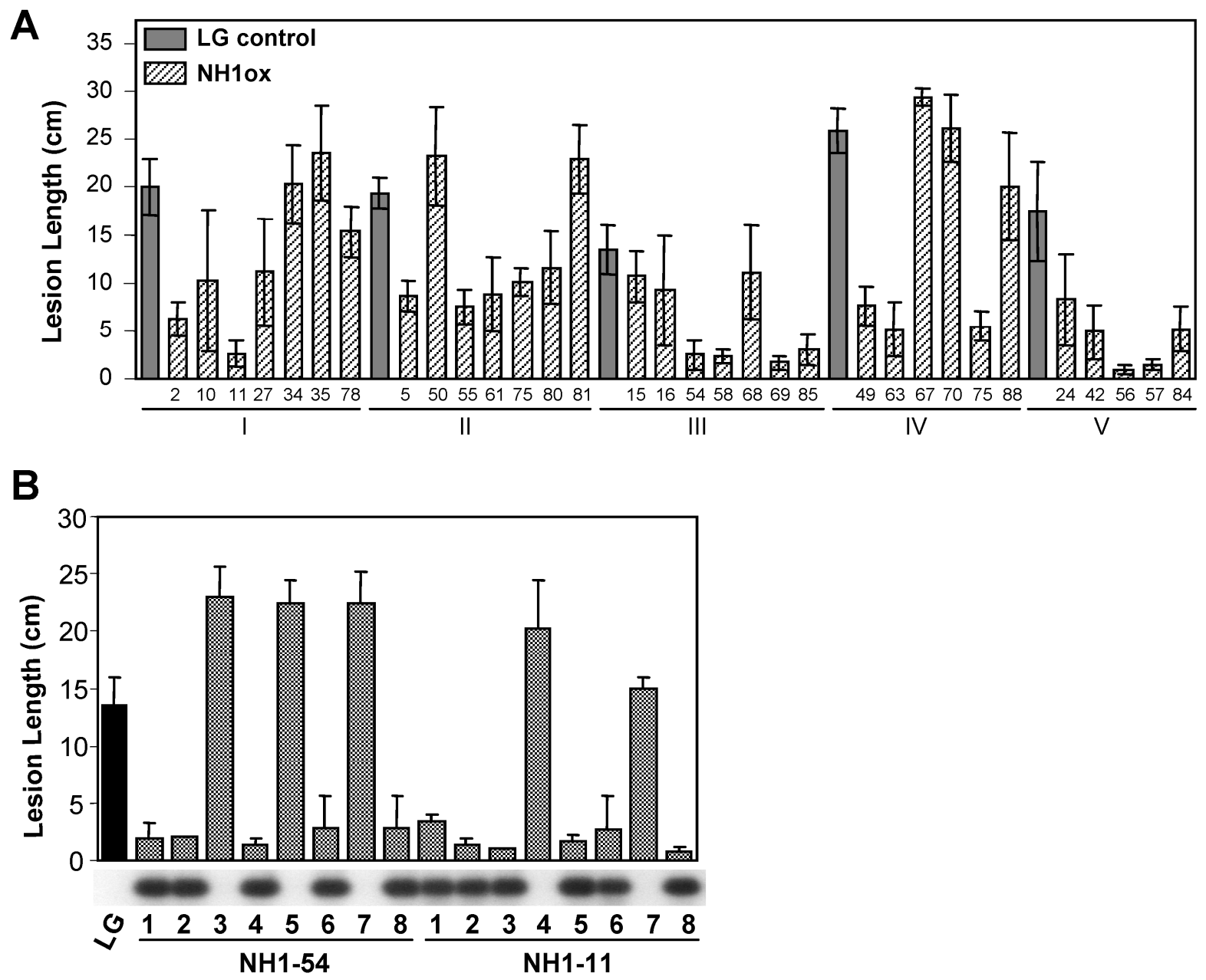

Fig. 2. Leaf lesion length of transgenic rice over-expressing NH1 (NH1ox) inoculated with Xanthomonas oryzae pv. oryzae Korean race 1 (KR1). A, Thirtytwo independently transformed 6-week-old T0 NH1ox transgenic plants and the LiaoGeng (LG) control were challenged with $X$. oryzae pv. oryzae in five separate inoculations (labeled I to V). B, Co-segregation of the Ubi-NHI transgene and the enhanced resistance phenotype. Lesion lengths of eight (labeled 1 to 8) segregating progeny from each of two lines (lines 11 and 54) are presented. Polymerase chain reaction (PCR) was performed using one primer specific to the ubiquitin promoter and the other specific to the NH1 cDNA. PCR results hybridized with a $N H 1$ probe are shown below the bar graph.
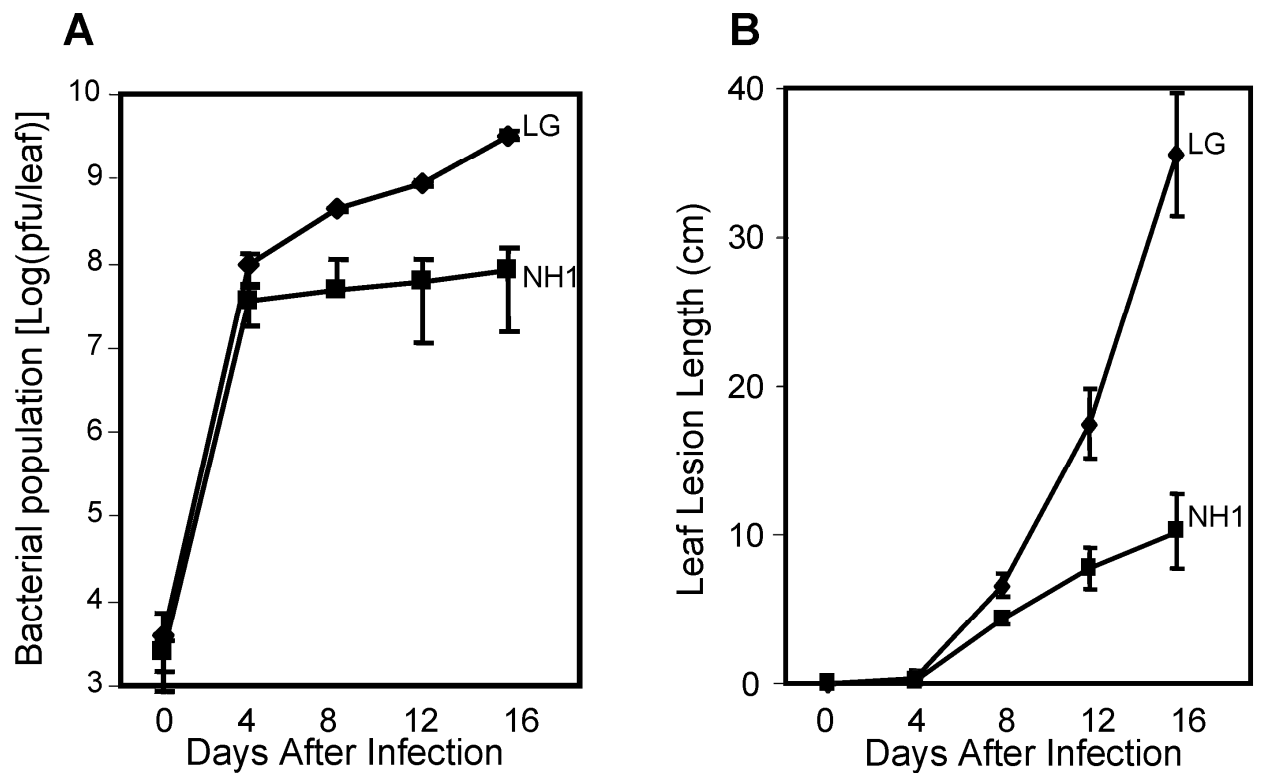

Fig. 3. Correlation of Xanthomonas oryzae pv. oryzae population growth and leaf lesion development. A, Bacterial growth curves. B, Leaf lesion development. Homozygous NH1ox transgenic plants (T2 progeny from line \#11) and LiaoGeng (LG) control plants were inoculated with X. oryzae pv. oryzae KR1. Four leaves each were collected from NH1ox or LG at day $0,4,8,12$, and 16 post inoculation. Lesion length for each individual leaf was measured; each leaf then was ground individually in water and plated out to assess $X$. oryzae pv. oryzae population. Each data point represents the average and standard deviation of four leaves. 
development phenotype in NH1ox plants is accompanied by a reduction in $X$. oryzae pv. oryzae growth.

\section{Activation of defense genes.}

In Arabidopsis, NPRI regulates expression of many $P R$ genes, such as $P R 1, P R 2$, and $P R 5$. Not only do $n p r l / n i m 1$ mutants abolish $P R$ gene expression after induction, but also overexpression of NPRI results in stronger induction of $P R$ genes by chemical inducers and pathogens. Although overexpression of Arabidopsis NPRl in rice also leads to enhanced resistance, it was not known whether defense genes are activated. We carried out Northern hybridizations to test whether defense genes are activated in the NH1ox rice plants. Total RNA samples were extracted from 6-week-old, greenhousegrown, untreated plants of two homozygous NH1ox lines

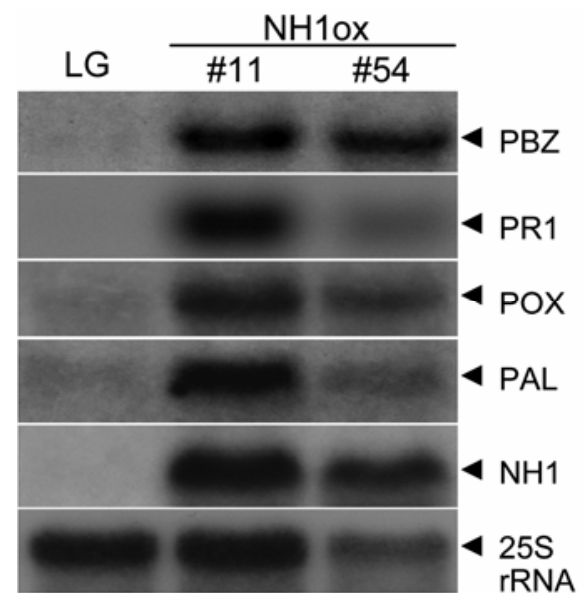

Fig. 4. RNA expression levels of $N H 1$ and defense-related genes of NH1ox lines 11 and 54 and the LiaoGeng (LG) control. Total RNA samples were sequentially probed with $P B Z 1, P A L, N H 1$, and $25 \mathrm{~S}$ rDNA, or with $P R 1, P O X, N H 1$, and $25 \mathrm{~S}$ rDNA. Total RNA $(10 \mu \mathrm{g})$ was loaded in each lane. (lines 11 and 54) and the LG control and hybridized with four defense-related genes: rice $P R 1 b, P B Z 1 / P R 10$ (Qi and Yang 1999), $P A L$ (phenylalanine ammonia lyase), and $P O X$ (peroxidase).

Northern analysis results are presented in Figure 4. First of all, in both NH1ox lines, the NH1 RNA levels were much higher than the endogenous level represented by the LG control. In fact, we have tested many of the NH1ox lines that display the resistance phenotype and all of them express high levels of NH1 mRNA. In LG, the four defense genes examined, especially $P B Z 1$ and $P R 1$, are expressed at very low levels. In contrast, all four defense-related genes obviously are constitutively expressed at elevated levels in the two NH1ox lines. These results demonstrate that overexpression of the $\mathrm{NH} 1$ gene in rice leads to constitutive expression of defense genes in the absence of induction by chemical or pathogen treatment.

\section{NH1ox plants develop lesion-mimic spots}

in the greenhouse and LMD spots in the growth chamber.

In a previous study, we observed that LMD spots develop on leaves of transgenic rice overexpressing Arabidopsis NPRI (NPR1ox) (Fitzgerald et al. 2004) when the plants were transferred from the greenhouse (high light conditions) to the growth chamber (approximately one-sixth light intensity, high humidity; details discussed below). Similarly, NH1ox plants develop LMD spots 3 weeks after transfer from the greenhouse to the growth chamber (Fig. 5A). These LMD spots normally start near the leaf tips and slowly move down the leaves. In severe cases, the entire leaf will senesce and die.

In addition to the LMD phenotype, we have observed a second lesion-mimic phenotype on the NH1ox but not the NPR1ox plants. Older leaves of the NH1ox plants spontaneously develop lesion-mimic spots in the greenhouse when the plants are 8 to 9 weeks old, at the preflowering (booting) stage. Two leaves from NH1ox plants displaying such typical lesion-mimic spots are shown in Figure 5B (indicated by the arrowhead). Leaves from LG plants are free of such spots. In contrast to the LMD phenotype, development of the lesion
A

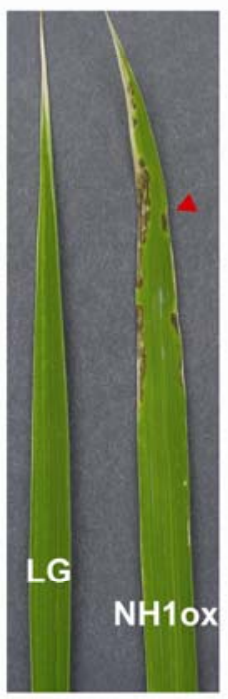

B

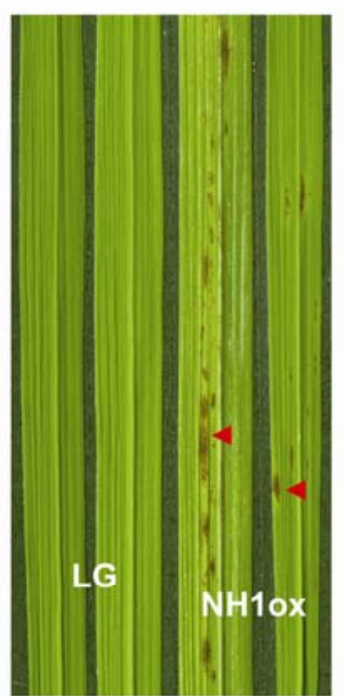

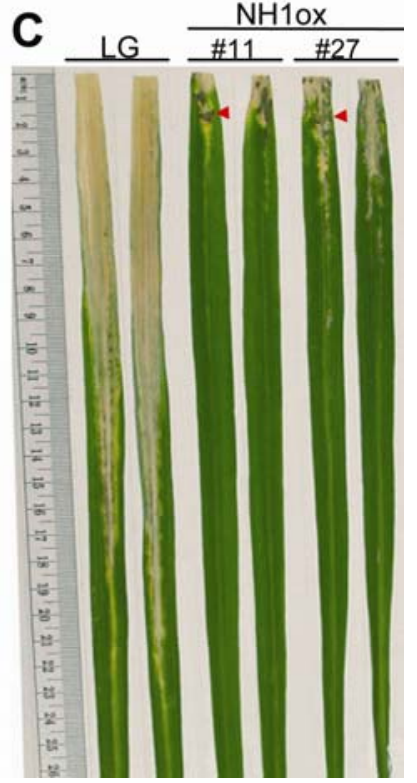

Fig. 5. Environment-induced lesion mimic or cell death (LMD), spontaneous lesion-mimic spots, and Xanthomonas oryzae pv. oryzae-induced hypersensitive response (HR)-like necrosis on NH1ox plants. A, Environment-induced LMD (marked by the arrowhead); 3-week-old plants grown in the greenhouse were transferred to the growth chamber and grown for another 3 weeks. B, Spontaneous, stage-dependent lesion-mimic spots. Leaves of 9-weekold NH1ox transgenic plants and the LiaoGeng (LG) control grown in the greenhouse. The arrowheads mark typical lesion-mimic spots. C, X. oryzae pv. oryzae-induced HR-like necrosis of NH1ox plants. Leaf tips were infected with $X$. oryzae pv. oryzae when plants were 6 weeks old. Pictures were taken 2 weeks after inoculation. The HR-like necrosis normally becomes visible 3 days after inoculation with $X$. oryzae pv. oryzae. 
mimic spots on the NH1ox rice in the greenhouse is growthstage dependent and its initiation is not restricted to the leaf tips. It occurs only on mature leaves of older plants and appears throughout the leaf. Nevertheless, NH1ox transgenic plants grow as well as the LG control in the greenhouse and show a seed set comparable to the control (data not shown).

Older leaves of NH1ox rice plants show a tendency to senesce precociously compared with wild-type plants. This tendency becomes more obvious after pathogen infection; although $X$. oryzae pv. oryzae-inoculated leaves of NH1ox plants develop shorter lesions, older leaves (below the inoculated ones) of the same plant display accelerated senescence (not shown).

In addition, NH1ox plants show more obvious HR-like response a few days after challenge with the $X$. oryzae pv. oryzae pathogen. Leaves of the NH1ox plants develop $X$. oryzae pv. oryzae-induced, HR-like necrotic spots (Fig. 5C, arrowheads) near the $X$. oryzae pv. oryzae inoculation sites, whereas fully susceptible leaves of the LG control show few spots. These results further support the conclusion that the NH1ox transgenic plants possess an altered defense response to $X$. oryzae pv. oryzae infection.

\section{Growth of NH1ox plants is more sensitive to light.}

When grown in the greenhouse, NH1ox plants show no obvious difference from the wild-type LG control in appearance and seed setting. However, when grown in the growth chamber, the NH1ox plants display retarded growth relative to the LG control. The difference in growth chamber-grown plants and the similarity in the greenhouse-grown plants at 3 weeks old is shown in Figure 6A. In addition, under the growth chamber conditions, older leaves of NH1ox plants tend to senesce precociously.

To quantify the results, plant height, fresh weight of aerial parts, number of tillers per plant, and leaf width were measured at 8 weeks old. The results summarized in Figure 6B show that, although greenhouse-grown NH1ox plants were essentially the

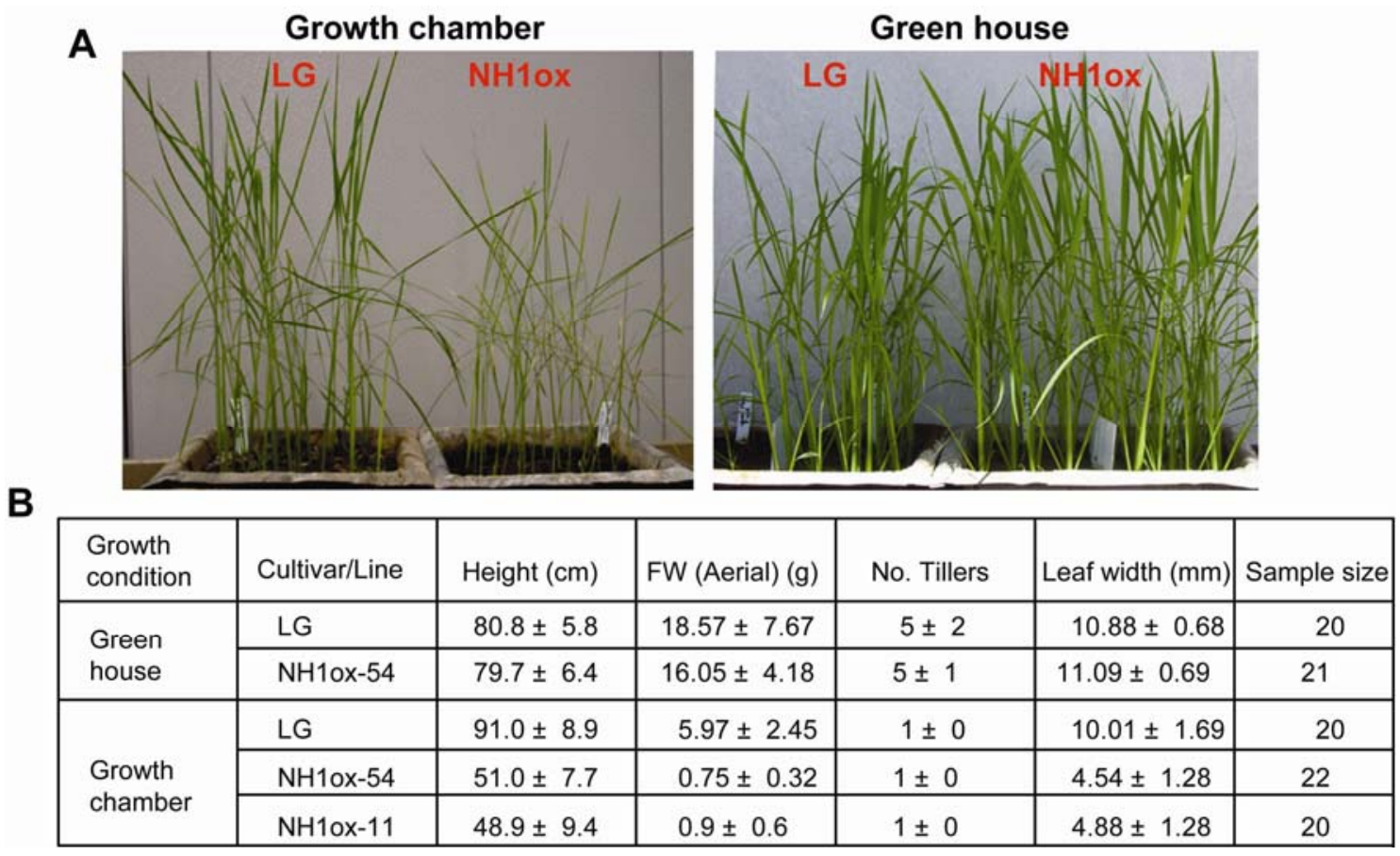

Fig. 6. Growth of NH1ox rice plants under greenhouse and growth chamber conditions. A, Approximately 30 homozygous $\mathrm{T} 2$ progeny from NH1ox transgenic lines 54 and 11 and the LiaoGeng (LG) control were grown either in the growth chamber or greenhouse. Pictures were taken when plants were 3 weeks old. B, At 8 weeks old, NH1ox and LG plants were harvested and measured for total height and fresh weight (FW) of the aerial portions of the plant, as well as tiller number and leaf width.
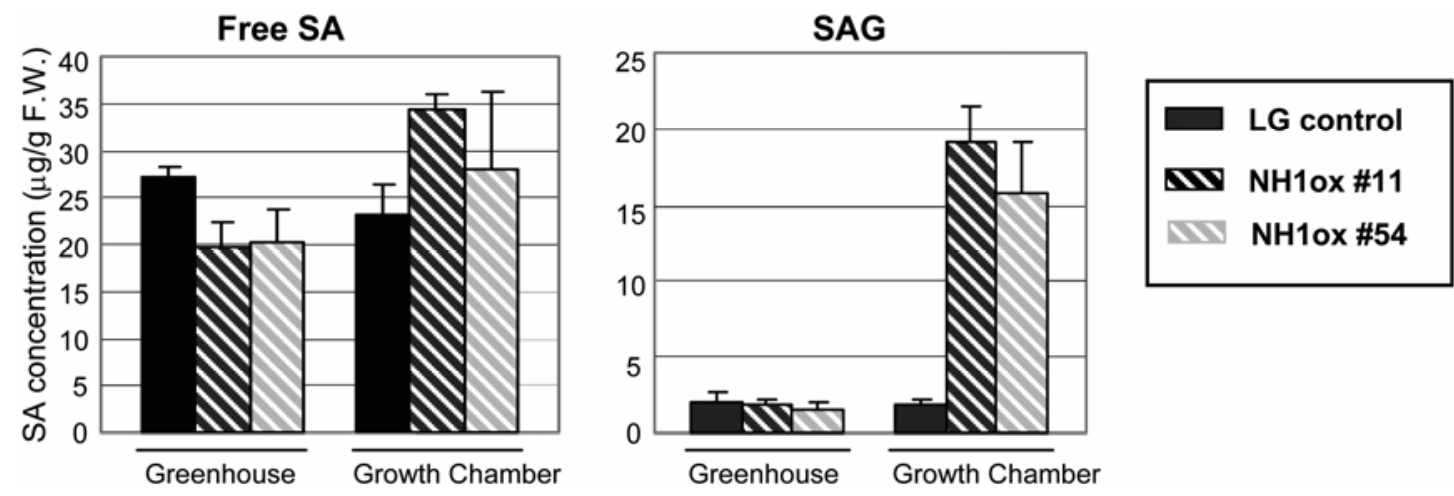

Fig. 7. Free and conjugated salicylic acid (SA) contents. The third leaf of each plant was harvested from 3-week-old LiaoGeng (LG) or NH1ox (lines 11 and 54) rice grown in the greenhouse or growth chamber. Two to three replicate samples per cultivar or line (approximately 15 plants per cultivar or line for each growth environment) were analyzed by high-performance liquid chromatography for both free-SA and glucose-conjugated SA (SAG) contents. Each bar represents the mean and standard deviation for the sample. 
same as the LG wild-type control, growth chamber-grown NH1ox plants (both lines 11 and 54) have much smaller statures $(2 x)$, lower fresh weights $(7 x)$, and narrower $(2 x)$ or smaller leaves. Under the growth chamber condition, both LG and NH1ox plants failed to develop additional tillers. These results indicate that the NH1ox rice plants are more sensitive to the growth chamber environment, which has a different light spectrum and lower light intensity than the greenhouse environment (Fitzgerald et al. 2004).

\section{NH1ox plants have altered levels of $S A$ contents.}

We previously reported that overexpression of Arabidopsis NPRI in rice correlated with reduced SA accumulation in leaves (Fitzgerald et al. 2004). To assess possible effects of overexpressing $\mathrm{NHI}$ on SA accumulation in rice, we measured SA levels. Both free SA and SA-glucoside (SAG) contents were determined for the third leaves of NH1ox (lines 11 and 54) and LG plants grown under greenhouse and growth chamber conditions. Free SA levels are lower in NH1ox lines than in the LG wild type under greenhouse conditions, similar to the observation for NPR1ox rice plants (Fig. 7). However, free SA levels are higher in NH1ox lines under the growth chamber condition. Moreover, under the growth chamber conditions, the SAG levels in both NH1ox lines are strikingly (more than $8 \times$ ) higher than the LG wild type. Although all the rice plants grown in the greenhouse contain low levels of SAG, the SAG levels of the two growth-chamber-grown NH1ox lines reached levels similar to free SA, suggesting that free SA might be converted to SAG when it reached a certain threshold level. In short, the NH1ox plants accumulate much higher total SA (free SA + SAG) than the wild type in the growth chamber, whereas they contain slightly lower total SA when grown in the greenhouse. Thus, SA accumulation appears more sensitive to environmental changes in the NH1ox plants compared with the LG controls.

\section{Interaction of rice NPR1 \\ with TGA transcription factors is conserved.}

The rice $\mathrm{NHI}$ was identified in two-hybrid screening by using an NPR1-interacting protein NRR rather than an rTGA2 protein. Therefore, we tested directly whether rice NH1 interacted with rTGA2.2 in the yeast two-hybrid assay. The rTGA2.2 protein was fused to the B42 activation domain, whereas each of $\mathrm{NH} 1$ and its mutants was fused to the LexA DNA binding domain. The wild-type NH1 interacted strongly with rTGA2.2, showing intense blue color, and the vector-only control displayed little blue color (Fig. 8A).

We also generated two NH1 point mutations at amino acids critical for NPR1 function and conserved with rice NH1 and tested their effects on interaction with rTGA2.2. Mutation $\mathrm{H}_{338} \mathrm{Y}(\mathrm{HY})$ corresponded to npr1-1 and mutation $\mathrm{C}_{150} \mathrm{Y}(\mathrm{CY})$ to npr1-2. Each of the mutations was capable of completely abolishing the interaction with rTGA2.2 (Fig. 8A). The Western results of yeast protein (extracted from yeast cell carrying these constructs) (Fig. 8B) show a slightly reduced protein level for the HY mutant. However, the slightly lower protein expression cannot account for the complete abolishment of interaction by the HY mutation. The CY mutant had a significantly reduced protein level. Thus, protein instability of $\mathrm{CY}$ may have contributed to the lack of interaction with rTGA2.2. Overall, the HY mutation had the same effect on NH1 as on NPR1 with regards to the interaction with rTGA2.2.

\section{DISCUSSION}

We have isolated two rice cDNA clones encoding NPR1 homologs by yeast two-hybrid screening. Our results showed that NH1 strongly interacted with rTGA2.2, a rice bZIP transcription factor similar to Arabidopsis TGA2; the interaction was abolished by $\mathrm{HY}$ and $\mathrm{CY}$ mutations corresponding to npr1-1 and npr1-2, respectively, suggesting that the NH1rTGA2.2 interaction is similar to that of NPR1-TGA2. To study $\mathrm{NH} 1$ function, we overexpressed $\mathrm{NH} 1$ in the rice cv. LG, which is highly susceptible to $X$. oryzae pv. oryzae KR1. Transgenic NH1ox plants show high levels of resistance when challenged with KR1. The resistance phenotype is heritable and correlates with the presence of the $\mathrm{Ubi}-\mathrm{NH} 1$ transgene. Bacterial growth curve analysis showed a correlation between the resistance phenotype and the reduction in $X$. oryzae pv. oryzae population in the NH1ox rice. These results are consistent with our previous report, which showed that overexpression of the Arabidopsis NPRl in rice enhances resistance to $X$. oryzae pv. oryzae and suggested that rice shares a resistance pathway similar to the NPRl-mediated pathway (Chern et al. 2001). Overexpression of rice $N H 1$ seems to be more effective in conferring resistance to $X$. oryzae pv. oryzae than overexpression of Arabidopsis NPRI because the NH1ox plants exhibit a strong enhancement in resistance when challenged with KR1. NPR1ox rice plants exhibited only very moderate resistance to this strain (data not shown). However, because the transgenic lines are in different genetic backgrounds, these results should be interpreted with caution.

RNA blot analysis indicated that the NH1ox rice plants constitutively express defense genes. This defense gene expression was independent of the presence of visible lesion mimic spots which normally occur at 8 weeks or older, because RNA samples taken from 6-week-old and younger (data not shown) greenhouse-grown plants showed elevated defense gene expression. In contrast, PR1 and PBZ1/PR10 were significantly expressed in NPR1ox plants only when LMD spots were visible after the plants were transferred to growth chambers. Thus, NH1ox plants appeared to activate the defense gene more readily than NPR1ox rice. The NH1ox results also contrasted with findings from Arabidopsis over-

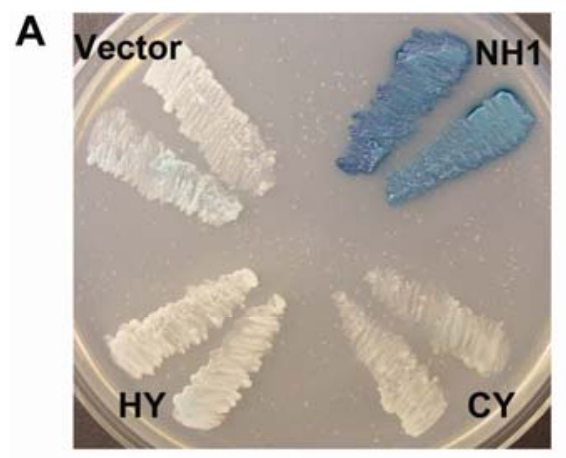

Bait: B42AD::rTGA2.2

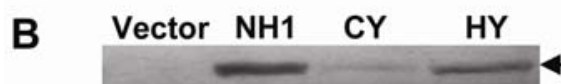

Fig. 8. Yeast two-hybrid assay for interaction of rTGA2.2 and NH1 wildtype and mutants. A, In-plate $\beta$-galactosidase activity assay. The rTGA2.2 protein was fused to the $\mathrm{B} 42$ activation domain while each of $\mathrm{NH} 1$ and its mutants was fused to the LexA DNA binding domain. NH1 mutant $\mathrm{C}_{150} \mathrm{Y}$ (CY) corresponds to the npr1-2 mutation and $\mathrm{H}_{338} \mathrm{Y}(\mathrm{HY})$ to the npr1-1 mutation. X-gal was included in the medium. The picture was taken 2 days after yeast cells were streaked on the plate. B, Western analysis of yeast protein. Protein samples were extracted from yeast cells expressing either wild-type NH1, mutant protein CY or HY fused to the LexA DNA binding domain, or the LexA domain alone. Protein samples were probed with an antibody (Clontech) against LexA. The arrowhead indicates the location of the fusion proteins. Approximately $150 \mu \mathrm{g}$ of protein were loaded in each lane. 
expressing NPR1, in which defense gene expression was not observed until induction by chemical or pathogen treatment (Cao et al. 1998). In short, our data showed a difference between rice and Arabidopsis in the regulation of defense gene induction, possibly due to the fact that rice contains much higher levels of endogenous SA than Arabidopsis (Silverman et al. 1995). Although the high levels of SA in rice may cause the overexpressed NH1 protein to be constitutively activated, the low levels of SA in Arabidopsis would keep overexpressed NPR1 protein inactive until SAR induction and SA synthesis to induce defense.

The development of lesion-mimic spots on leaves of NH1ox plants at preflowering stage in the greenhouse indicated that the defense pathway or pathways was activated, consistent with the Northern results. However, we did not observe any obvious detrimental developmental effects in the NH1ox plants, probably because the rice plants already were at a productive stage when the lesion mimic spots occurred. We also did not find significant reduction in seed set of the NH1ox plants, although large-scale experiments would be needed to draw a more definitive conclusion.

The spontaneous and age-dependent development of the lesion-mimic spots on NH1ox plants was distinct from the LMD spots that we observed for both NPR1ox and NH1ox rice plants. Transgenic NPR1ox and NH1ox rice lines did not exhibit LMD in the greenhouse; the LMD spots developed only when the plants were transferred from the greenhouse to growth chambers (low light). The LMD spots normally start near the leaf tips and slowly move down the leaves in severe cases (Fitzgerald et al. 2004). In contrast, initiation of the lesion mimic spots on the NH1ox rice was not restricted to the leaf tips but occurred throughout the leaves. Although the LMD spots can develop on young plants, development of the lesion-mimic spots on NH1ox rice in the greenhouse was limited strictly to plants approximately 8 weeks old and older. These results also suggest a difference in the rice $\mathrm{NH} 1$ and Arabidopsis NPR1 proteins. Like the spontaneous induction of defense genes, development of the lesion-mimic spots may be due, in part, to the high endogenous SA levels in rice.

SA is involved in leaf senescence in Arabidopsis. SA levels are increased fourfold in senescent leaves compared with mature green ones. Senescence-enhanced genes, such as LSC94 and LSC460, were induced by SA application. Upregulation of these genes at senescence was inhibited in $n p r l$ and pad4 mutants and $N a h G$ transgenic Arabidopsis; changes in gene expression were accompanied by a delayed yellowing and reduced necrosis (Morris et al. 2000). Thus, SA levels and NPR1 both are involved in senescence-associated gene expression in Arabidopsis.
Older leaves of NH1ox rice plants show a tendency to senesce early when compared with wild-type plants. This tendency becomes more obvious after pathogen infection. Although $X$. oryzae pv. oryzae-inoculated leaves of NH1ox plants develop shorter lesions, older leaves (below the inoculated ones) of the same plant experience accelerated senescence. This phenomenon is reminiscent of INA-treated Arabidopsis plants, where wild-type Arabidopsis experience early senescence in the lower leaves approximately a week after the treatment, whereas nprl mutants remain green and unchanged (unpublished data). This suggests that $\mathrm{NH} 1$ in rice most likely is involved in a SAR-like response and elevated levels of NH1 may augment the response.

Interestingly, NH1ox plants display growth retardation when grown in growth chambers, indicating that they are more sensitive to the growth-chamber light conditions. We compared the wavelength spectrum and intensity of light in our growth chamber and greenhouse. Growth chambers evidently had much lower light intensity (approximately one-sixth of the greenhouse) and very different light quality or wavelength spectrum from the greenhouse (Fitzgerald et al. 2004). Thus, it is likely that suboptimal light quality and low light intensity may cause the growth retardation effects of NH1ox plants. In Arabidopsis, it has been reported that phytochrome signaling modulates a SA-perceptive pathway (Genoud et al. 2002). The induction of $P R l$ by SA is dependent on $p h y A$ - and phyB-controlled light signaling pathways; in darkness as well as dim light, SA-induced $P R$ gene expression and the HR to pathogens are strongly reduced.

In contrast to many other plants, rice normally contains more free-SA than SAG (Fitzgerald et al. 2004; Silverman et al. 1995). The growth-chamber-grown NH1ox plants accumulated much higher SAG and slightly higher free SA than the wild type, whereas greenhouse-grown NH1ox plants contained slightly lower free SA and SAG levels. These results suggest that NH1 may be involved in SA signaling and in the regulation of SA content in response to environmental changes (Fig. 9). The light quality and intensity may be the environmental factor that $\mathrm{NH} 1$ responds to, leading to changes in SA levels. The strikingly higher levels of SAG in the growth-chambergrown NH1ox plants indicated that, under low light conditions, NH1 may activate SA-conjugating enzymes, leading to much higher SAG levels. A possible reason that leaves of NH1ox plants have such high free SA contents in the growth chamber is that NH1ox plants might be undergoing senescence. Support for this hypothesis is the observation that leaves of growth-chamber-grown NH1ox plants displayed LMD symptoms resembling senescence. The LMD symptoms
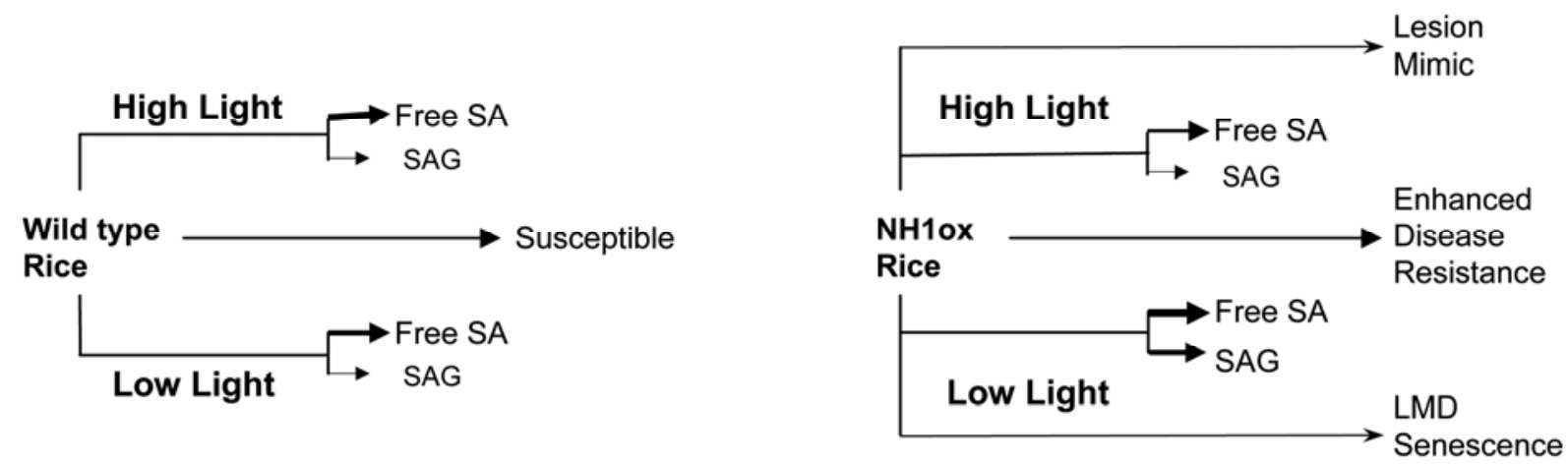

Fig. 9. Schematic representation of the modulation of free salicylic acid (SA) and glucose-conjugated SA (SAG) accumulation by the light intensity in wildtype (left panel) and NH1ox (right panel) rice plants and effects on lesion mimic and disease resistance. High light is represented by greenhouse conditions and low light by growth chamber conditions. NH1ox represents high levels of NH1 expression. Levels of free SA and SAG accumulation are depicted by the different sizes of the arrows; thicker arrows indicate higher levels. 
on NH1ox plants induced by the growth chamber environment were similar to the LMD observed for NPR1ox plants under the same environment. However, NPR1ox plants did not accumulate high SA under this environment.

In summary, the study of rice $\mathrm{NHI}$ has revealed similarities as well as differences between rice and Arabidopsis with regard to the defense responses and plant growth. It appears that, although rice and Arabidopsis share conserved defense pathways, the regulation of these pathways and the links to other plant pathways may be quite divergent. Therefore, it is important to directly study $\mathrm{NH} 1$ in rice in order to understand its involvement in rice defense and other biological pathways.

\section{MATERIALS AND METHODS}

\section{Plant materials and growth conditions.}

Rice (Oryzae sativa L.) plants were maintained in the greenhouse, which had a photosynthetically active radiation (PAR) value equal to $674 \mu \mathrm{mol} \mathrm{m} \mathrm{m}^{-2} \mathrm{~s}^{-1}$ in spring. The growth chamber had a light intensity PAR equivalent to $103 \mu \mathrm{mol} \mathrm{m} \mathrm{m}^{-2} \mathrm{~s}^{-1}$, set on a 14-h daytime period and a temperature cycle of 28 and $26^{\circ} \mathrm{C}$, $90 \%$ humidity. For X. oryzae pv. oryzae inoculation, rice plants were grown in the greenhouse until they were 6 weeks old and transferred to a growth chamber. The $X$. oryzae pv. oryzae strain KR1 was used to inoculate rice by the scissorsdip method (Kauffman et al. 1973).

\section{Rice transformation.}

Rice cv. LG was used as the recipient for transformation. Agrobacterium EHA105 was used to infect rice callus for transformation. Rice transformation protocol was as described before (Chern et al. 2001).

\section{Plasmid construction.}

For rice transformation, a 1.95-kb full-length $\mathrm{NHI}$ cDNA fragment was PCR amplified from the original yeast two-hybrid pAD-Gal4 clone using primers prNH13 (AAATCTAGAGGAT CCCAATGGAGCCGCCGACCA) and prNH6 (CCTCGAGTA CAAGCACTA). The NHI PCR product was cloned into pBluescript II SK- and its sequence confirmed by sequencing. The full-length NHI cDNA insert was excised by cutting with BamHI and SpeI and cloned into binary vector Ubi-C1300, digested with the same enzymes, creating plasmid UbiNH1/C1300. The Ubi-C1300 binary vector was generated by cloning a maize ubiquitin promoter (Christensen and Quail 1996) and a nos3' fragment into the Cambia 1300 vector by the same way as described before (Chern et al. 2001) for UbiC1301.

To create the LexA fusion protein for the yeast two-hybrid test, a 2-kb, full-length $N H I$ cDNA was excised with EcoRI and XhoI and cloned into plasmid pNLex digested by EcoRI and SalI enzymes. The $\mathrm{C}_{150} \mathrm{Y}$ mutant was generated by using primers prNH8 (GTCCTCGTCGACGTAGAGGCACGCCGC CT) and SS020 (AGGGATGTTTAATACCACTAC) to amplify a $0.5-\mathrm{kb} 5^{\prime}$ end of $\mathrm{NH1}$ cDNA; the $0.5-\mathrm{kb}$ CY fragment was cut with EcoRI and SalI and ligated to a $1.5-\mathrm{kb}$ SalI-XhoI fragment using the unique SalI site to assemble the full-length cDNA. To create mutant $\mathrm{H}_{338} \mathrm{Y}$, a $3^{\prime}$ end was amplified using primers prNH9 (ACTGTTCTTTACATTGCTGCGAGGCG AA) and prNH6. A middle piece of $\mathrm{NH} 1$ was amplified with prNH10 (GCAGCAATGTAAAGAACAGTATAACCTCTTG) and prNH16 (AACTCGAGATCTACGAGGCGCTGCGGCT GGT). The two pieces were annealed together and the $1.5-\mathrm{kb}$ fragment amplified with prNH16 and prNH6. The 1.5-kb HY fragment was joined with a $0.5-\mathrm{kb} \mathrm{NH1} \mathrm{5'}$ end fragment at the unique NotI site to assemble the full-length cDNA. All PCR products were confirmed by DNA sequencing. The full-length
CY and HY mutants were cloned into the pNLex vector using the EcoRI and XhoI sites the same way as with the wild-type NHI cDNA.

\section{PCR, DNA, and RNA blot hybridization.}

Rice genomic DNA extraction was done according to a protocol described previously (Dellaporta et al. 1984). PCR of the Ubi-NH1 transgene was carried out with the maize ubiquitin promoter-specific primer Ubi-1 (TGATATACTTGGATGATG GCA) and $\mathrm{NH} 1$-specific primer prNH22 (GGACGGCGATG CGCGCGTC). DNA and RNA blotting and hybridization was performed as described before (Chern et al. 2001). For the rice $P R I b$ probe, a $1.3-\mathrm{kb}$ fragment of $P R I b$ was amplified from IR24 genomic DNA using primers osPR1-1 (AAGAATTCAA GTCCTGCGTACAAATC) and osPR1-2 (AAGAATTCTAGA GAAGTGCGGCGATG). The PCR product was cut with EcoRI and cloned into pBluescript II SK-; the sequence of the insert was verified by DNA sequencing. The rice $P B Z / P R 10$ probe was the same as described before (Fitzgerald et al. 2004).

\section{SA measurement.}

The measurement of SA contents of rice leaf tissues was done as described previously (Fitzgerald et al. 2004).

\section{ACKNOWLEDGMENTS}

This work was supported by National Institute of Health grant GM55962 and National Science Foundation grant 0096901 awarded to P. C. Ronald. We thank M. Whalen for critical reading of the manuscript and helpful suggestions.

\section{LITERATURE CITED}

Cao, H., Glazebrook, J., Clarke, J., Volko, S., and Dong, X. 1997. The Arabidopsis nprl gene that controls systemic acquired resistance encodes a novel protein containing ankyrin repeats. Cell 88:57-63.

Cao, H., Bowling, S. A., Gordon, A. S., and Dong, X. 1994. Characterization of an Arabidopsis mutant that is nonresponsive to inducers of systemic acquired resistance. Plant Cell 6:1583-1592.

Cao, H., Li, X., and Dong, X. 1998. Generation of broad-spectrum disease resistance by overexpression of an essential regulatory gene in systemic acquired resistance. Proc. Natl. Acad. Sci. U.S.A. 95:6531-6536.

Chern, M.-S., Fitzgerald, H. A., Yadav, R. C., Canlas, P. E., Dong, X., and Ronald, P. C. 2001. Evidence for a disease-resistance pathway in rice similar to the NPR1-mediated signaling pathway in Arabidopsis. Plant J. 27:101-113.

Christensen, A. H., and Quail, P. H. 1996. Ubiquitin promoter-based vectors for high-level expression of selectable and/or screenable marker genes in monocotyledonous plants. Transgenic Res. 5:213-218.

Clarke, S. M., Mur, L. A., Wood, J. E., and Scott, I. M. 2004. Salicylic acid dependent signaling promotes basal thermotolerance but is not essential for acquired thermotolerance in Arabidopsis thaliana. Plant J. 38:432-447.

Delaney, T. P., Friedrich, L., and Ryals, J. A. 1995. Arabidopsis signal transduction mutant defective in chemically and biologically induced disease resistance. Proc. Natl. Acad. Sci. U.S.A. 92:6602-6606.

Dellaporta, S. L., Wood, J., and Hicks, J. B. 1984. Pages 36-37 in: Molecular Biology of Plants. A Laboratory Course Manual. M. Russell, ed. Cold Spring Harbor Laboratory, Cold Spring Harbor, NY, U.S.A.

Despres, C., DeLong, C., Glaze, S., Liu, E., and Fobert, P. 2000. The Arabidopsis npr1/nim 1 protein enhances the DNA binding activity of a subgroup of the TGA family of bZIP transcription factors. Plant Cell 12:279-290.

Dong, X. 1998. SA, JA, ethylene, and disease resistance in plants. Curr. Opin. Plant Biol. 1:316-323.

Fan, W., and Dong X. 2002. In vivo interaction between NPR1 and transcription factor TGA2 leads to salicylic acid-mediated gene activation in Arabidopsis. Plant Cell 14:1377-1389.

Fitzgerald, H. A., Chern, M.-S., Navarre, R., and Ronald, P. C. 2004 Overexpression of (At)NPR1 in rice leads to a BTH- and environmentinduced lesion-mimic/cell death phenotype. Mol. Plant-Microbe Interact. 17:140-151.

Friedrich, L., Lawton, K., Dietrich, R., Willitis, M., Cade, R., and Ryals, J. 2001. NIM1 overexpression in Arabidopsis potentiates plant disease re- 
sistance and results in enhanced effectiveness of fungicides. Mol. PlantMicrobe Interact. 9:1114-1124.

Friedrich, L., Lawton, K., Ruess, W., Masner, P, Speckner, N., Gt Rella, M., Meier, B., Dinher, S., Staub, T., Uknes, S., Metraux, J.-P., Kessman, H., and Ryals, J. 1996. A benzothiadiazole derivative induces systemic acquired resistance in tobacco. Plant J. 9:61-70.

Genoud, T., Buchala, A. J. Chua, N.-H., and Metraux, J.-P. 2002. Phytochrome signaling modulates the SA-perceptive pathway in Arabidopsis. Plant J. 31:87-95.

Glazebrook, J. 2001. Genes controlling expression of defense responses in Arabidopsis: 2001 status. Curr. Opin. Plant Biol. 4:301-308.

Glazebrook, J., Rogers, E. E., and Ausubel, F. M. 1996. Isolation of Arabidopsis mutants with enhanced disease susceptibility by direct screening. Genetics 143:973-982,

Gorlach, J., Volrath, S., Knauf-Beiter, G., Hengy, G., Beckhove, U., Kogel, K.-H., Oostendorp, M., Staub, T., Ward, E., Kessmann, H., and Ryals, J. 1996. Benzothiadiazole, a novel class of inducers of systemic acquired resistance, activates gene expression and disease resistance in wheat. Plant Cell 8:629-643.

Johnson, C., Boden, E., and Arias, J. 2003. Salicylic acid and NPR1 induce the recruitment of trans-activating TGA factors to a defense gene promoter in Arabidopsis. Plant Cell 15:1846-1858.

Kauffman, H. E., Reddy, A. P. K., Hsieh, S. P. V., and Marca, S. D. 1973. An improved technique for evaluation of resistance of rice varieties to Xanthomonas oryzae. Plant Dis. Rep. 57:537-541.

Kinkema, M., Fan, W., and Dong, X. 2000. Nuclear localization of NPR1 is required for activation of PR gene expression. Plant Cell 12:23392350.

Kunkel, B. N., and Brooks, D. M. 2002. Cross talk between signaling pathways in pathogen defense. Curr. Opin. Plant Biol. 5:325-331.

Li, J., Brader, G., and Palva, E. T. 2004. The WRKY70 transcription factor: a node of convergence for jasmonate-mediated and salicylate-mediated signals in plant defense. Plant Cell 16:319-331.

Liu, Y., Schiff, M., Marathe, R., and Dinesh-Kumar, S. P. 2002. Tobacco Rar1, EDS1 and NPR1/NIM1 like genes are required for N-mediated resistance to tobacco mosaic virus. Plant J. 30:415-429.

Lorrain, S., Valileau, F., Balague, C., and Roby, D. 2003. Lesion mimic mutants: keys for deciphering cell death and defense pathways in plants. Trends Plant Sci. 8:263-271.

Morris, S. W., Vernoolij, B., Titatarn, S., Starrett, M., Thomas, S., Wiltse, C. C., Frederiksen, R. A., Bhandhufalck, A., Hulbert, S., and Uknes, S. 1998. Induced resistance responses in maize. Mol. Plant-Microbe Interact. 11:643-658.

Morris, K., Mackerness, S. A., Page, T., John, C. F., Murphy, A. M., Carr, J. P., Buchanan-Wollaston, V. 2000. Salicylic acid has a role in regulating gene expression during leaf senescence. Plant J. 23:677685 .

Mou, Z., Fan, W., and Dong, X. 2003. Inducers of plant systemic acquired resistance regulate NPR1 function through redox changes. Cell 113:110.

Mysore, K. S., and Ryu, C. M. 2004. Nonhost resistance: how much do we know? Trends Plant Sci. 9:97-104.

Pieterse, C. M. J., Van Wees, S. C. M., Van Pelt, J. A., Knoester, M., Laan, R., Gerrits, H., Weisbeek, P. J., and Van Loon, L. C. 1998. A novel sig- naling pathway controlling induced systemic resistance in Arabidopsis. Plant Cell 10:1571-1580.

Qi, M., and Yang, Y. 1999. Differential expression of rice $P R-1$ and $P R-10$ genes induced by blast fungus, elicitor, and chemical treatments. (Abstr.) Phytopathology 89:S62

Rohilla, R., Singh, U. S., and Singh, R. L. 2002. Mode of action of aciebnzolar- $S$-methyl against sheath blight of rice caused by Rhizoctonia solani Kuhn. Pest Manage. Sci. 58:63-69.

Ryals, J. A., Neuenschwander, U. H., Willits, M. G., Molina, A., Steiner, H.-Y., and Hunt, M. D. 1996. Systemic acquired resistance. Plant Cell 8:1809-1819.

Ryals, J., Weymann, K., Lawton, K., Friedrich, L., Ellis, D., Steiner, H.-Y., Johnson, J., Delaney, T. P., Jesse, T., Vos, P., and Uknes, S. 1997. The Arabidopsis NIM1 protein shows homology to the mammalian transcription factor inhibitor IкB. Plant Cell 9:425-439.

Schweizer, P., Schlagenhauf, E., Schaffrath, U., and Dudler, R. 1999. Different patterns of host genes are induced in rice by Pseudomonas syringae, a biological inducer of resistance, and the chemical inducer benzothiadiazole (BTH). Eur. J. Plant Pathol. 105:659-665.

Shah, J., Tsui., F., and Klessig, D. F. 1997. Characterization of a salicylic acid-insensitive mutant (sail) of Arabidopsis thaliana, identified in a selective screen utilizing the SA-inducible expression of the tms 2 gene. Mol. Plant Microbe Interact. 10:69-78.

Silverman, P., Seskar, M., Kanter, D., Schweizer, P., Metraux, J.-P., and Raskin, I. 1995. Salicylic acid in rice: biosynthesis, conjugation, and possible role. Plant Physiol. 108:633-639.

Smith, J. A., and Metraux, J.-P. 1991. Pseudomonas syringae pathovar syringae induces systemic resistance to Pyricularia oryzae in rice. Physiol. Mol. Plant Pathol. 39:451-461.

Spoel, S. H., Koornneef, A., Claessens, S. M. C., Korzelius, J. P., Van Pelt, J. A. Mueller, M. J., Buchala, A. J., Metraux, J.-P., Brown, R., Kazan, K., Van Loon, L. C., Dong, X., and Pieterse, C. M. J. 2003. NPR1 modulates cross-talk between salicylate- and jasmonate-dependent defense pathways through a novel function in the cytosol. Plant Cell 15:760-770.

Turner, J. G., Ellis, C., and Devoto, A. 2002. The jasmonate signal pathway. Plant Cell 14:s153-s164.

Yin, Z., Chen, J., Zeng, L., Goh, M., Leung, H., Khush, G., and Wang, G.L. 2000. Characterizing rice lesion mimic mutants and identifying a mutant with broad-spectrum resistance to rice blast and bacterial blight. Mol. Plant-Microbe Interact. 13:869-876.

Zhang, Y., Fan, W., Kinkema, M., Li, X., and Dong, X. 1999. Interaction of NPR1 with basic leucine zipper protein transcription factors that bind sequences required for salicylic acid induction of the PR-1 gene. Proc. Natl. Acad. Sci. U.S.A. 96:6523-6528.

Zhang, Y., Tessaro, M. J., Lassner, M., and Li, X. 2003. Knockout analysis of Arabidopsis transcription factors TGA2, TGA5, and TGA6 reveals their redundant and essential roles in systemic acquired resistance. Plant Cell 15:2647-2653.

Zhou, J. M., Trifa, Y., Silva, H., Pontier, D., Lam, E., Shah, J., and Klessig, D. 2000. NPR1 differentially interacts with members of the TGA/OBF family of transcription factors that bind an element of the PR-1 gene required for induction by salicylic acid. Mol. Plant-Microbe Interact. 13:191-202. 